\title{
IncRNA MALAT1 modulates cancer stem cell properties of liver cancer cells by regulating YAP1 expression via miR-375 sponging
}

\author{
LIYING ZHAO ${ }^{1 *}$, GUOHUA LOU ${ }^{2 *}$, AICHUN LI $^{2}$ and YANNING LIU ${ }^{2}$ \\ ${ }^{1}$ Department of Infectious Disease, The Second Affiliated Hospital, Zhejiang University School of Medicine, \\ Hangzhou, Zhejiang 310009; ${ }^{2}$ State Key Laboratory for Diagnosis and Treatment of Infectious Diseases, \\ National Clinical Research Center for Infectious Diseases, Collaborative Innovation Center for Diagnosis and \\ Treatment of Infectious Diseases, The First Affiliated Hospital of School of Medicine, \\ Zhejiang University, Hangzhou, Zhejiang 310003, P.R. China
}

Received November 9, 2019; Accepted April 8, 2020

DOI: $10.3892 / \mathrm{mmr} .2020 .11196$

\begin{abstract}
Liver cancer stem cells (CSCs) are functionally defined by their ability to undergo self-renewal, and may contribute to metastasis, recurrence and drug resistance in liver cancer. The long non-coding RNA metastasis-associated lung adenocarcinoma transcript 1 (MALAT1) has been implicated in tumor formation and metastasis of liver cancer. However, the exact mechanism by which MALAT1 modulates liver CSC features remains largely unknown. In the present study, the expression level of MALAT1 was elevated in cancer spheroids compared with the corresponding levels noted in parental liver cancer cells, whereas the suppression of MALAT1 resulted in markedly reduced sphere formation and decreased expression of stemness factors in liver cancer cells. Dual-luciferase assay and RNA pull-down assays further indicated an interaction between MALAT1 and microRNA (miR)-375, and identified Yes-associated protein 1 (YAP1) as a direct target of miR-375 in liver cancer cells. In addition, YAP1 expression was correlated with MALAT1 in liver cancer. The reduced expression of YAP1 caused by knockdown of MALAT1 with MALAT1 small interfering RNA (si-MALAT1) could be partially abolished by miR-375 inhibition, suggesting that MALAT1 may regulate YAP1 expression by sponging miR-375. Furthermore, YAP1 overexpression rescued the decrease in CSC features of liver cancer cells caused by si-MALAT1, further supporting
\end{abstract}

Correspondence to: Dr Yanning Liu, State Key Laboratory for Diagnosis and Treatment of Infectious Diseases, National Clinical Research Center for Infectious Diseases, Collaborative Innovation Center for Diagnosis and Treatment of Infectious Diseases, The First Affiliated Hospital of School of Medicine, Zhejiang University, 79 Qingchun Road, Hangzhou, Zhejiang 310003, P.R. China E-mail: liuyanning@zju.edu.cn

*Contributed equally

Key words: liver cancer, cancer stem cell, metastasis-associated lung adenocarcinoma transcript 1, microRNA-375, Yes-associated protein 1 that MALAT1-mediated YAP1 signaling was required for the stem-like characteristics of liver CSCs. The present study revealed that MALAT1 may promote CSC properties of liver cancer cells by upregulating YAP1 expression via sponging miR-375. The MALAT1/miR-375/YAP1 axis may serve as a novel target for liver cancer therapy, particularly for the eradication of liver CSCs.

\section{Introduction}

Primary hepatic cancer is the third leading cause of cancer-associated mortality worldwide, and $\sim 90 \%$ of primary hepatic cases are hepatocellular carcinoma (1). Surgical resection and liver transplantation are the first-line treatments used for liver cancer therapy. However, only $10-20 \%$ of patients with liver cancer are surgical candidates. Although liver-directed locoregional therapy, systemic chemotherapy or molecular therapy, such as sorafenib administration, are used for liver cancer therapy, these treatment strategies provide limited success due to high cancer cell heterogeneity (2). Cancer stem cells (CSCs) are a small population of cancer cells exhibiting self-renewal and differentiation abilities (3). CSCs are involved in tumor initiation, tumor metastasis and recurrence, and drug resistance (4). Liver CSCs can be identified by specific surface markers, such as epithelial cell adhesion molecule, CD133, CD44, CD90, CD13 and CD24 (5-7). However, the exact biological function of liver CSCs remains unclear. The investigation of the molecular mechanisms critical to the generation of liver CSC populations may provide novel therapeutic strategies for liver cancer.

Since the development of the Human Genome Project, long non-coding RNAs (lncRNAs), which are non-protein coding RNA transcripts $>200$ nucleotides, have attracted considerable attention. Although lncRNAs were initially believed to be transcriptional noise, subsequent studies have demonstrated that they play functional roles in various physiological and pathological processes, including carcinogenesis, tumor metastasis and drug resistance $(8,9)$. However, the mechanism by which lncRNAs modulate liver CSC features remains largely unknown. Metastasis-associated lung adenocarcinoma transcript 1 (MALAT1) is a ubiquitously expressed and highly conserved IncRNA, which has been demonstrated to be 
markedly overexpressed in several human malignancies (10). The expression of MALAT1 has been reported to be associated with clinical parameters and cancer prognosis (10). MALAT1 may also be involved in tumor growth, metastasis and drug resistance of liver cancer $(11,12)$, and has been reported to be associated with the CSC features of pancreatic cancer (13) and glioma (14). These findings suggest that MALAT1 can modulate stemness in liver cancer cells.

In the present study, MALAT1 was demonstrated to be a regulator of liver CSCs by effecting the expression levels of Yes-associated protein 1 (YAP1), a major transcriptional effector of the Hippo pathway. MALAT1 performed these functions by acting as a competitive endogenous RNA (ceRNA) for microRNA (miRNA/miR)-375. The MALAT1/miR-375/YAP1 axis may provide a potential target for liver cancer therapy and could be used to develop novel therapeutic drugs against liver CSCs.

\section{Materials and methods}

Patients and clinical tissues. Fresh liver tumor specimens and matched non-tumor liver samples were obtained from 20 patients (15 male patients and 5 female patients; age, 35-71 years) with hepatocellular carcinoma (without other malignant tumors) during hepatic resection at The Second Affiliated Hospital, Zhejiang University (Hangzhou, China) between January 2017 and October 2019. The present study was approved by the Hospital Ethics Committee (ethics no. 2019-099) and all patients signed informed consent for their participation in the study. The samples were immediately snap-frozen in liquid nitrogen.

Cell culture. The human liver cancer cell lines Huh7, Hep3B, HepG2 and 293T cells were cultured in DMEM (Gibco; Thermo Fisher Scientific, Inc.) containing 10\% fetal bovine serum (Gibco; Thermo Fisher Scientific, Inc.), $100 \mathrm{U} / \mathrm{ml}$ penicillin and $100 \mu \mathrm{g} / \mathrm{ml}$ streptomycin (Gibco; Thermo Fisher Scientific, Inc.), and were maintained at $37^{\circ} \mathrm{C}$ in a humidified atmosphere containing $5 \% \mathrm{CO}_{2}$. Cell line authentication was performed by genetic profiling using polymorphic short tandem repeat loci.

MALAT1 expression analysis by reverse transcription-quantitative $P C R$ ( $R T-q P C R)$. Total RNA was prepared from liver tumor tissues and cancer cells using TRIzol ${ }^{\circledR}$ reagent (Invitrogen; Thermo Fisher Scientific, Inc.). A PrimeScript ${ }^{\mathrm{TM}}$ RT Reagent kit with genomic DNA Eraser (Takara Bio, Inc.) was used to reverse transcribe RNA into cDNA at $42^{\circ} \mathrm{C}$ for $1 \mathrm{~h}$ according to the manufacturer's instructions. Subsequently, qPCR was performed using an ABI Prism 7900 kit (Applied Biosystems; Thermo Fisher Scientific, Inc.). The following thermocycling conditions were used for qPCR: Initial denaturation at $95^{\circ} \mathrm{C}$ for $30 \mathrm{sec}$; followed by 40 cycles at $95^{\circ} \mathrm{C}$ for $5 \mathrm{sec}$ and at $60^{\circ} \mathrm{C}$ for $30 \mathrm{sec}$. The following primers were used for qPCR: MALAT1 forward, 5'-ATACCTAACCAGGCATAACA-3' and reverse, 5'-AGTAGACCAACTAAGCGAAT-3'; and GAPDH forward, 5'-GACCTGACCTGCCGTCTAG-3' and reverse, 5'-AGGAGTGGGTGTCGCTGT-3'. The $2^{-\Delta \Delta C q}$ method was used to determine relative MALAT1 expression levels (15), which were normalized to the internal reference gene GAPDH.
Sphere formation assay. The cells $\left(1 \times 10^{3}\right.$ cells $\left./ \mathrm{ml}\right)$ were seeded on ultralow attachment culture dishes (Corning Inc.) in DMEM/F12 serum-free medium (Gibco; Thermo Fisher Scientific, Inc.) supplemented with $10 \mathrm{ng} / \mathrm{ml}$ fibroblast growth factor-2 (Peprotech, Inc.), $20 \mathrm{ng} / \mathrm{ml}$ epithelial growth factor (Peprotech, Inc.), N2 (Thermo Fisher Scientific, Inc.), B27 (Thermo Fisher Scientific, Inc.), 1\% sodium pyruvate (Thermo Fisher Scientific, Inc.), 2 mM L-glutamine (Thermo Fisher Scientific, Inc.) and penicillin/streptomycin. After 2 weeks of incubation at $37^{\circ} \mathrm{C}$, the spheres with a size $>100 \mu \mathrm{m}$ were counted and images were obtained using a light stereomicroscope (Olympus Corporation).

Plasmid construction. The fragments of wild-type MALAT1 and of the 3'-UTR of wild-type YAP1 containing the putative binding site of miR-375, as well as their corresponding mutant sequences, which were generated using a Site-Directed Mutagenesis Kit (cat. no. E0554S; New England Biolabs, Inc.), were synthetized and cloned into a pmirGLO vector (Promega Corporation) using the $\mathrm{XhoI}$ and Not I sites in order to generate wild-type MALAT1 reporter (MALAT1-WT), mutant MALAT1 reporter (MALAT1-MUT), wild-type YAP1 reporter (YAP1-WT) or mutant YAP1 reporter (YAP1-MUT) dual-luciferase reporter plasmids. To assess the function of YAP1, the full-length sequence of YAP1 was ligated into the pcDNA 3.1 plasmid (Thermo Fisher Scientific, Inc.) to construct a YAP1 overexpression plasmid (oeYAP1). An empty pcDNA3.1 plasmid was used as a control (oeCtrl). All constructs were established using the In-Fusion ${ }^{\circledR}$ Clone kit (Clontech Laboratories, Inc.).

Transfection of small interfering RNA (siRNA), miRNA and plasmids. siRNA sequences targeting MALAT1 expression (si-MALAT1; cat. no. siB170613115806-1-5) and control siRNA sequences (si-Ctrl; siN0000002-1-5), miR-375 mimic (sense, 5'-UUUGUUCGUUCGGCUCGCGUGA-3' and antisense, 5'-UCACGCGAGCCGAACGAACAAA-3'), miRNA mimic negative control (miR-NC, sense, 5'-UUUGUACUACACAAA AGUACUG-3' and antisense, 5'-CAGUACUUUUGUGUAG UACAAA-3'), miR-375 inhibitor (5'-UCACGCGAGCCGAAC GAACAAA-3'), and miRNA inhibitor negative control (miR-NC, 5'-CAGUACUUUUGUGUAGUACAAA-3') were purchased from Guangzhou RiboBio Co., Ltd. Lipofectamine ${ }^{\circledR} 3000$ (Invitrogen; Thermo Fisher Scientific, Inc.) was used for siRNA and plasmid transfection, and Lipofectamine ${ }^{\mathrm{TM}}$ RNAiMAX (Invitrogen; Thermo Fisher Scientific, Inc.) was used for miRNA transfection. At $75 \%$ confluence, cells were transfected with siRNA $(100 \mathrm{~nm})$, miRNA $(50 \mathrm{nM})$ or plasmid $(1 \mu \mathrm{g} / \mathrm{ml})$ at $37^{\circ} \mathrm{C}$. At $24 \mathrm{~h}$ post-transfection, MALAT1 expression in siRNA-transfected cells and miR-375 expression in miRNA mimic- or miRNA inhibitor-transfected cells were assessed using RT-qPCR analysis, and YAP1 expression in plasmid-transfected cells was assessed using Western blotting (Fig. S1).

Dual-luciferase assay. MiRcode (version 11; www. mircode.org) and TargetScan (version 7.2; www.targetscan. org/vert_72) were used for predicting the potential target sequences between MALAT1 and miR-375 and between miR-375 and YAP1, respectively. The dual-luciferase reporter plasmids MALAT1-WT, MALAT1-MUT, YAP1-WT or 
A
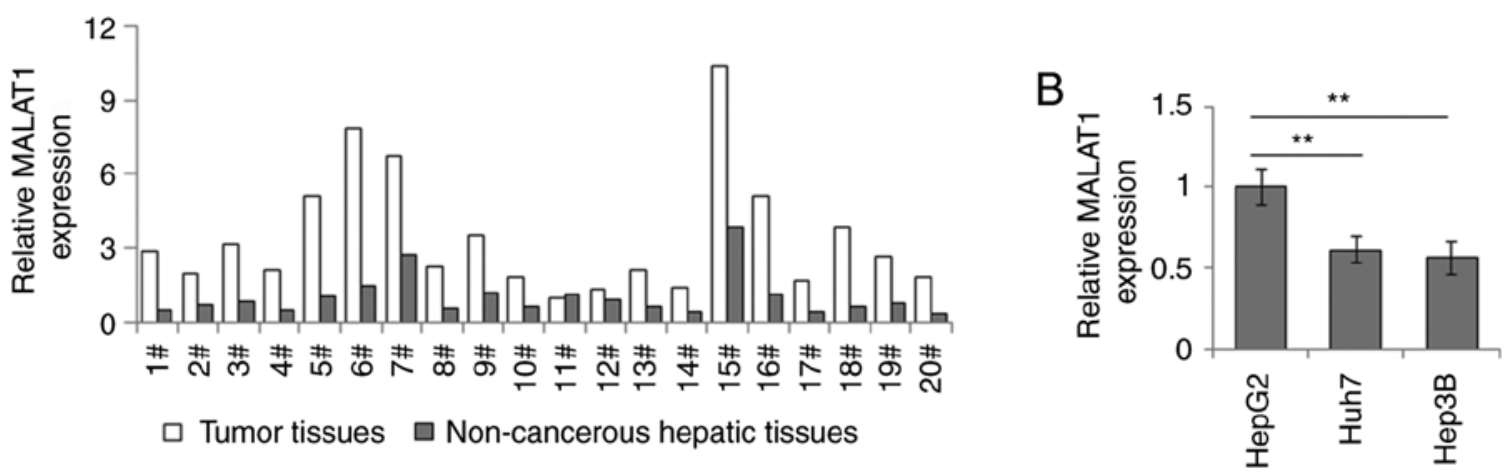

Figure 1. MALAT1 is overexpressed in liver cancer. (A) RT-qPCR analysis of MALAT1 expression in liver tumor tissues and paired non-cancerous hepatic tissues. (B) Relative expression of MALAT1 in three liver cancer cell lines was detected by RT-qPCR. Data are presented as the mean \pm SD. ${ }^{* *} \mathrm{P}<0.01, \mathrm{n}=3$. MALAT1, metastasis-associated lung adenocarcinoma transcript 1; RT-qPCR, reverse transcription-quantitative PCR.

YAP1-MUT $(1 \mu \mathrm{g} / \mathrm{ml})$, and miR-375 mimics or miRNA mimic negative control $(50 \mathrm{nM})$ were co-transfected into $293 \mathrm{~T}$ cells $\left(5 \times 10^{4}\right.$ cells/well) using Lipofectamine ${ }^{\circledR} 3000$ (Invitrogen; Thermo Fisher Scientific, Inc.) at $37^{\circ} \mathrm{C}$. A total of $48 \mathrm{~h}$ post-transfection, the Renilla and Firefly luciferase activities were measured using a Dual-Luciferase Reporter Assay system (Promega Corporation) according to manufacturer's protocol. The results were quantified by the ratio of the activity of Renilla luciferase to that of Firefly luciferase.

RNA pull-down assay. Biotinylated miR-375 was labeled using the RNA 3' End Desthiobiotinylation Kit (Pierce; Thermo Fisher Scientific, Inc.) according to manufacturer's protocol. RNA pull-down assays were performed following transfection of biotin-labeled-miR-375 into liver cancer cells $\left(1 \times 10^{6}\right.$ cells/well). Three sequences were used as follows: 3'-biotin-labeled miR-375 (bio-miR-375-WT), 3'-biotin-labeled miR-375 with a mutation at the binding site between MALAT1 and miR-375 (bio-miR-375-MUT), and 3'-biotin-labeled miR-NC (bio-miR-NC). The cells were harvested after $48 \mathrm{~h}$ of transfection at $37^{\circ} \mathrm{C}$ and subsequently lysed in RNase-free cell lysis solution (1 Mm HEPES, $200 \mathrm{mM} \mathrm{NaCl}, 1 \%$ Triton X-100, $10 \mathrm{mM} \mathrm{MgCl}_{2}, 200 \mathrm{U} / \mathrm{ml}$ RNase Inhibitor) at $4^{\circ} \mathrm{C}$. Streptavidin agarose beads (Pierce; Thermo Fisher Scientific, Inc.) were incubated with the cell lysis overnight at $4^{\circ} \mathrm{C}$ for pulling down the biotin-labeled miRNA and associated RNAs from the solution. After washing and centrifugation $(1,500 \mathrm{x} \mathrm{g}$; $10 \mathrm{~min}, 4^{\circ} \mathrm{C}$ ), the pellet was lysed with TRIzol ${ }^{\circledast}$. Subsequently, the expression levels of MALAT1 in the pull-down samples were measured by RT-qPCR.

Verteporfin treatment. Verteporfin (Sigma-Aldrich; Merck KGaA) was dissolved in DMSO and added to the cell medium $\left(1 \times 10^{3}\right.$ cells $\left./ \mathrm{ml}\right)$ for a final concentration of $10 \mu \mathrm{g} / \mathrm{ml}$ for $24 \mathrm{~h}$ at $37^{\circ} \mathrm{C}$. Equal concentration of DMSO was used as control.

Western blot analysis. A RIPA peptide lysis buffer (Beyotime Institute of Biotechnology) containing $1 \%$ protease inhibitor (Pierce; Thermo Fisher Scientific, Inc.) was used for tissue and cell lysis. Total protein was quantified using a bicinchoninic acid assay kit (Thermo Fisher Scientific, Inc.). Proteins (30 $\mu \mathrm{g} / \mathrm{lane})$ were separated via $10 \%$ SDS-PAGE and transferred to a $0.22-\mu \mathrm{m}$
PVDF membrane. After blocking with 5\% non-fat milk for $1 \mathrm{~h}$ at room temperature, the membranes were incubated overnight at $4^{\circ} \mathrm{C}$ with the following primary antibodies: anti-YAP1 $(1: 1,000$; cat.no.ab52771; Abcam), anti- phosphorylated (p)-YAP1 (1:1,000; cat. no. ab172374; Abcam), anti-c-Myc (1:1,000; cat. no. ab32072; Abcam), anti-Oct4 (1:1,000; cat. no. ab181557; Abcam), anti-Sox2 (1:1,000; cat. no. ab92494; Abcam) and anti-GAPDH (1:3,000; cat. no. 5174S; Cell Signaling Technology). Membranes were incubated with primary antibodies at room temperature for $3 \mathrm{~h}$. A horseradish peroxidase-conjugated goat anti-rabbit IgG H\&L (1:3,000; cat. no. ab7090; Abcam) was used as the secondary antibody and was used to incubate the membranes at room temperature for $1 \mathrm{~h}$. The protein bands were visualized using an Enhanced Chemiluminescence system (PerkinElmer Inc.) and detected using the ChemiScope Western Blot Imaging system (Clinx Science Instruments Co., Ltd.). ImageJ software (version 1.46; National Institutes of Health) was used for semi-quantification of the results.

Statistical analysis. Each experiment was repeated at least three times. The differences between two groups were compared using the independent samples t-test. One-way ANOVA followed by Bonferroni post-hoc test was used to determine significant differences between multiple groups. The correlation between YAP1 and MALAT1 was determined using Spearman correlation tests. The data were presented as mean $\pm \mathrm{SD}$. $\mathrm{P}<0.05$ was considered to indicate a statistically significant difference.

\section{Results}

MALAT1 is overexpressed in liver cancer. The RT-qPCR results indicated that MALAT1 expression was markedly higher in liver tumor tissues than that in the corresponding non-cancerous hepatic tissues (Fig. 1A). Moreover, the expression levels of lncRNA MALAT1 in the hepatoblastoma cell line HepG2 were higher compared with those in the common hepatoma cell lines Huh7 and Hep3B (Fig. 1B). These data indicated that MALAT1 expression was upregulated in liver cancer, especially in hepatoblastoma cells.

MALAT1 modulates CSC features of liver cancer cells. The role of MALAT1 in modulating the stemness of liver CSCs was 
A

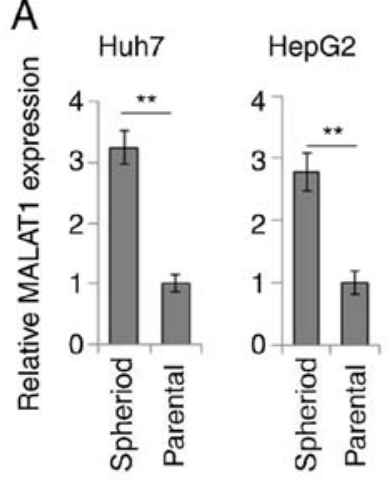

C

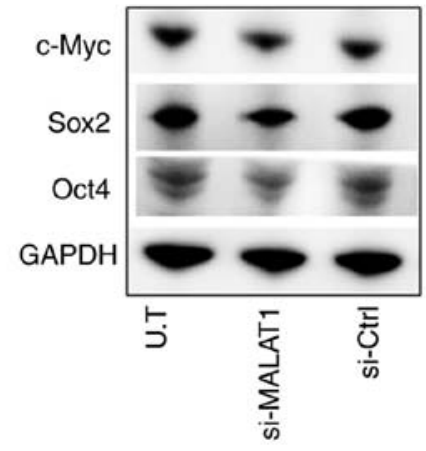

B

Huh7
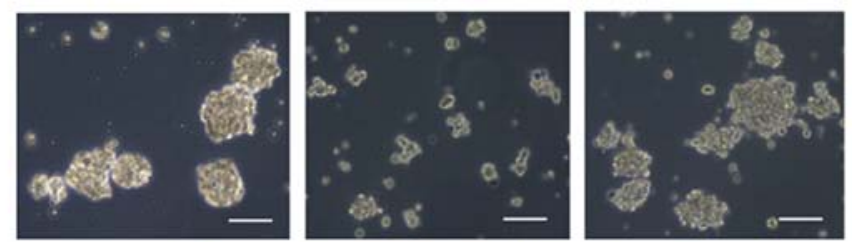

HepG2

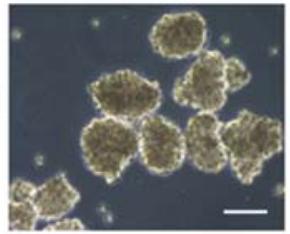

U T

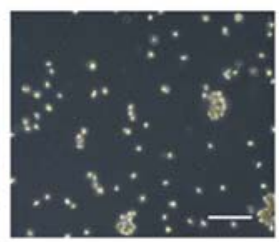

Si-MALAT1

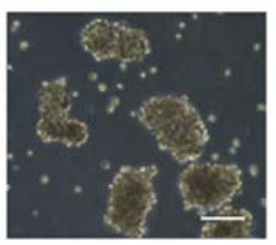

si-Ctrl

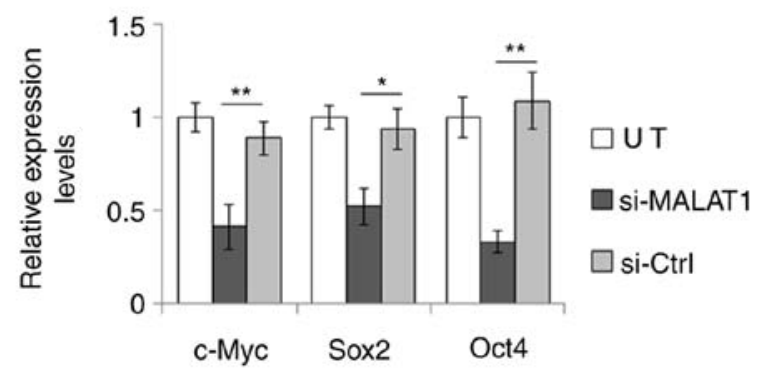

Figure 2. MALAT1 expression is associated with CSC features of liver cancer cells. (A) Reverse transcription-quantitative PCR detection of MALAT1 expression levels in spheroids and their parental cells. (B) Sphere formation assay on self-renewal capacities of si-MALAT1- and si-Ctrl-transfected liver cancer cells. Scale bar, $100 \mu \mathrm{m}$. (C) Western blot analysis and gray value assay of the expression levels of stemness factors in HepG2 cells transfected with si-MALAT1 or si-Ctrl. Data are presented as the mean \pm SD. ${ }^{*} \mathrm{P}<0.05,{ }^{* *} \mathrm{P}<0.01, \mathrm{n}=3$. MALAT1, metastasis-associated lung adenocarcinoma transcript 1 ; si-, small interfering RNA; CSC, cancer stem cells; UT, untreated control cells.

A

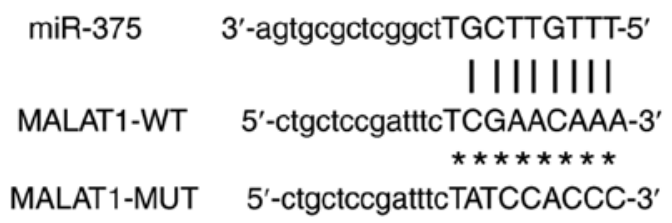

B

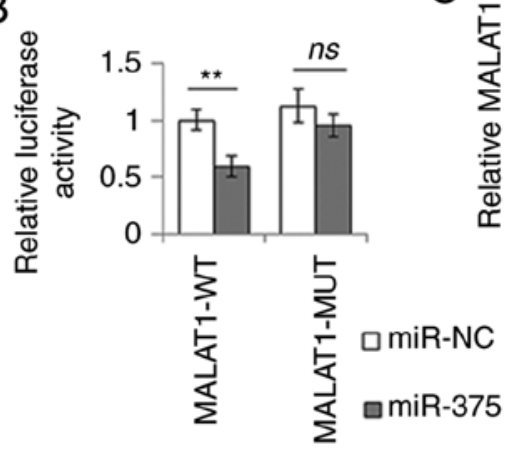

C

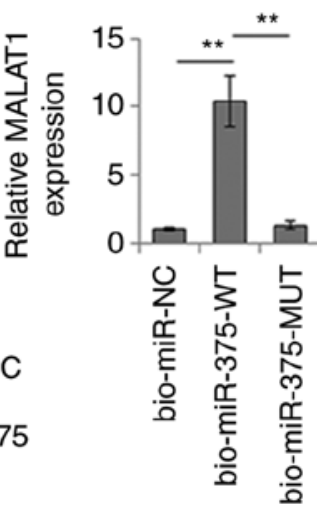

Figure 3. MALAT1 is a molecular sponge for miR-375. (A) Base-pairing between MALAT1 and miR-375. (B) Dual-luciferase activity of the WT and MUT MALAT1 reporters in the presence of miR-375 or miR-NC mimics in 293T cells. (C) RNA pull-down assay for detection of the interaction between MALAT1 and miR-375 in Huh7 cells. Data are presented as the mean $\pm \mathrm{SD} .{ }^{* *} \mathrm{P}<0.01, \mathrm{n}=3$. MALAT1, metastasis-associated lung adenocarcinoma transcript 1; MUT, mutant; WT, wild-type; miR, microRNA; NC, negative control; ns, not significant; bio, 3'-biotin.

examined. MALAT1 was highly expressed in spheroids derived from Huh7 and HepG2 cells (Fig. 2A). siRNA-induced downregulation of MALAT1 expression levels (Fig. S1) resulted in considerably suppressed sphere formation and reduced sphere size of the Huh7 and HepG2 liver cancer cell lines (Fig. 2B). In HepG2 cells, MALAT1 suppression significantly reduced the expression levels of c-Myc, Sox 2 and Oct4 (Fig. 2C), which are key transcription factors that modulate cancer cell stemness (16-18). These results indicated that MALAT1 could promote the stem-like properties of liver cancer cells.
MALAT1 interacts with miR-375. IncRNAs can act as miRNA ceRNAs. To confirm the role of MALAT1 in modulating CSC features of liver cancer cells as a ceRNA molecule, bioinformatics analysis was performed. The data led to the identification of several miRNA-binding sites on MALAT1. Among these miRNAs, miR-375 has previously been shown to be associated with the CSC phenotype (19). Therefore, the present study focused on miR-375 and further investigated the interaction between MALAT1 and miR-375 by dual-luciferase assay. miR-375 mimics significantly reduced the luciferase 
A

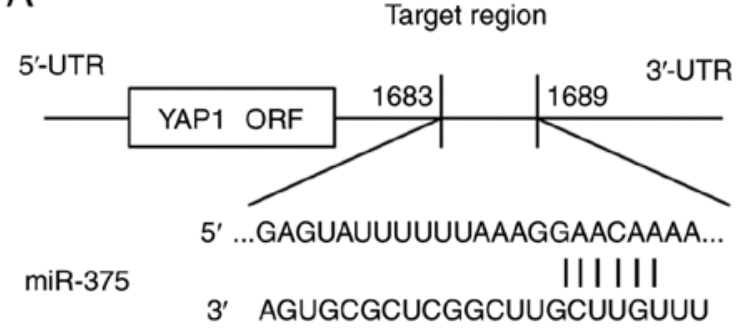

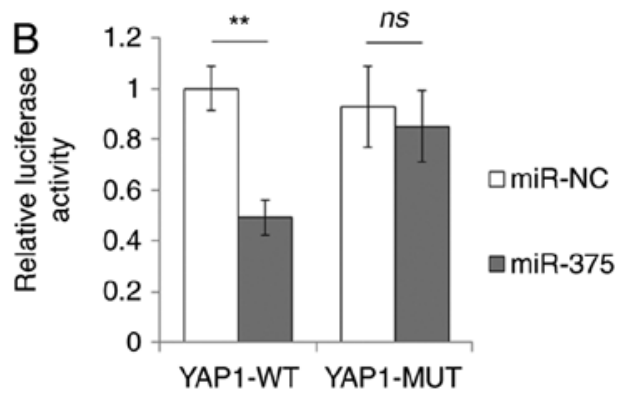

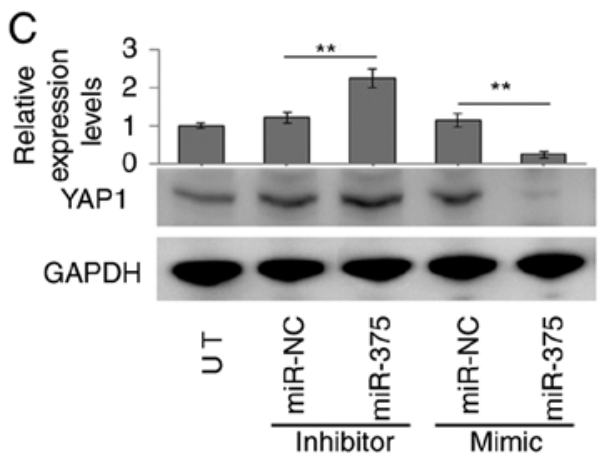

Figure 4. miR-375 directly targets YAP1. (A) Target site of miR-375 in YAP1 3'-UTR. (B) Dual-luciferase activity of the WT and MUT YAP1 3'-UTR reporters in the presence of miR-375 or miR-NC mimics in 293T cells. (C) Western blot analysis and gray value assay of YAP1 expression in Huh7 cells transfected with miR-375 mimics, miR-375 inhibitors, and their corresponding controls (miR-NC). Data are presented as the mean $\pm \mathrm{SD}$. ${ }^{* *} \mathrm{P}<0.01$, $\mathrm{n}=3$. UT, untreated control cells; ns, not significant; YAP1, Yes-associated protein 1; WT, wild-type; MUT, mutant; miR, microRNA; UTR, untranslated region; NC, negative control; ORF, open reading frame.

activity of MALAT1-WT compared with that of miR-NC, whereas no change was observed in the luciferase activity of MALAT1-MUT, which had a mutation in the miR-375 binding site (Fig. 3A and B). Subsequently, the biotin-streptavidin RNA pull-down assay was used to further determine the interaction of MALAT1 with miR-375. As expected, miR-375 could successfully pull down MALAT1. However, the mutation in the binding site of miR-375 to MALAT1 disturbed the pull-down of MALAT1 by this miRNA (Fig. 3C). These data confirmed the interaction between MALAT1 and miR-375, and suggested that MALAT1 may regulate liver CSC features by sponging miR-375.

YAPl is a direct target of miR-375. As miRNAs are known to regulate gene expression at a post-transcriptional level by interacting with the 3'-UTR of target genes, TargetScan was employed to predict the targets of miR-375. YAP1 is a major transcriptional effector for regulating liver CSC self-renewal capacity (20). In the present study, YAP1 was identified as a candidate target of miR-375 (Fig. 4A).

To verify whether YAP1 was an actual target of miR-375, the 3'-UTR fraction of YAP1 containing the predicted miR-375 binding site was cloned into a pmirGLO vector for YAP1-WT reporter construction. The dual-luciferase report assay indicated that miR-375 significantly reduced the luciferase activity of YAP1-WT compared with that of miR-NC. However, mutation of the miR-375 binding site of YAP1 3'-UTR (YAP1-MUT) abrogated the inhibitory effects of miR-375 (Fig. 4B).

The miR-375-mediated regulation of YAP1 expression was further examined in liver cancer cells. miR-375 mimic transfection significantly reduced YAP1 protein levels, whereas
miR-375 inhibitor transfection increased YAP1 expression in Huh7 cells (Fig. 4C). Together, these data strongly suggested that YAP1 was a direct target of miR-375.

MALAT1 regulates YAP1 expression by sponging miR-375. To further support the ceRNA hypothesis and investigate the regulatory mechanism of MALAT1 on YAP1 expression, YAP1 levels were measured in si-MALAT1-transfected Huh7 cells by Western blot analysis. As shown in Fig. 5A, the YAP1 expression levels were notably decreased in Huh7 cells transfected with si-MALAT1, which is similar to the finding in cells transfected with miR-375 mimic (Fig. 4C). In addition, si-MALAT1 caused a reduction in the expression levels of YAP1 that could be partially abolished by transfection with the miR-375 inhibitor (Fig. 5B). The association between the expression levels of YAP1 and MALAT1 was further determined in liver cancer cell lines and tumor samples. As shown in Fig. 5C, the highest YAP1 expression was detected in HepG2 cells, which also exhibited the highest level of MALAT1 expression compared with the Huh7 and Hep3B cell lines (Fig. 1B). Moreover, YAP1 expression, alongside MALAT1, was markedly increased in liver tumor tissues compared with in the corresponding non-cancerous hepatic tissues (Fig. 5E and F). A Spearman correlation analysis also determined that there was a positive correlation between YAP1 expression and MALAT1 expression in liver tumor tissues (Fig. 5D). These results indicated that MALAT1 could regulate YAP1 expression by sponging miR-375.

MALAT1-mediated upregulation of YAP1 affects the CSC features of liver cancer cells. YAP1 is ubiquitously activated in human malignancies and its expression is associated with the 
A

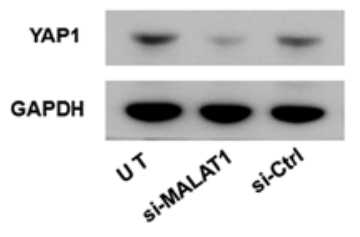

B

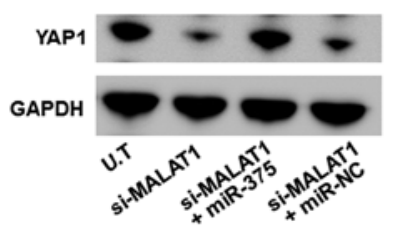

C

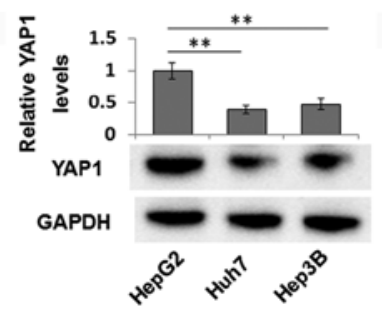

D

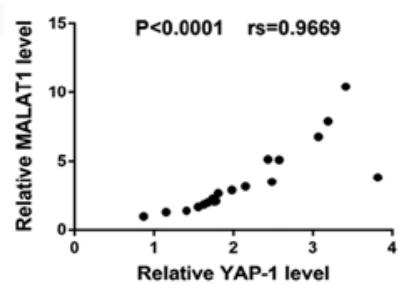

E

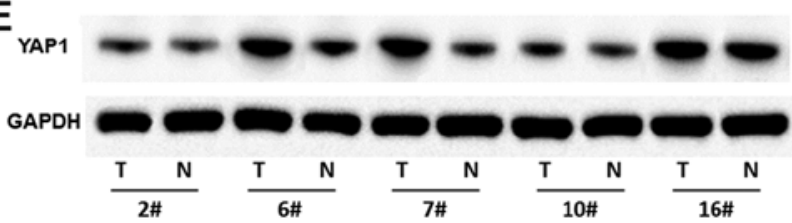

$\mathbf{F}$

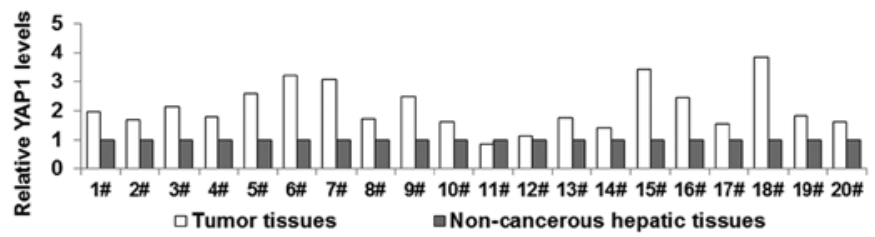

Figure 5. MALAT1 can regulate YAP1 expression by sponging miR-375. Western blot analysis of (A) YAP1 expression in the Huh7 cells transfected with si-MALAT1 or si-Ctrl, and (B) in the Huh7 cells co-transfected with si-MALAT1 and miR-375 inhibitor or si-MALAT1 and control inhibitor (miR-NC). (C) Western blot analysis of YAP1 expression in three liver cancer cell lines. (D) Spearman correlation analysis of MALAT1 and miR-375 expression levels in tumor samples. YAP1 expression levels in the T and paired $\mathrm{N}$ samples were detected by (E) Western blot analysis and subsequently (F) semi-quantified, relative to GAPDH. Data are presented as the mean $\pm \mathrm{SD} .^{* *} \mathrm{P}<0.01, \mathrm{n}=3$. MALAT1, metastasis-associated lung adenocarcinoma transcript 1 ; YAP1, Yes-associated protein 1; miR, microRNA; si-, small interfering RNA; NC, negative control; T, hepatic tumor tissues; N, non-cancerous hepatic tissues; UT, untreated control cells.

A

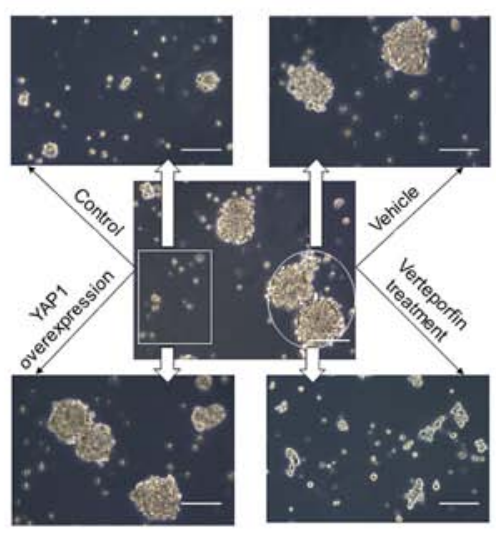

B

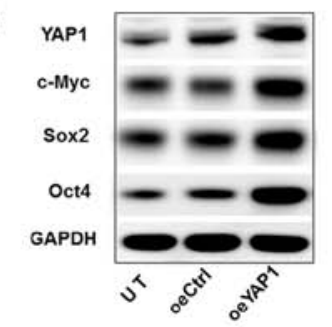

D

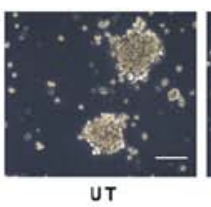

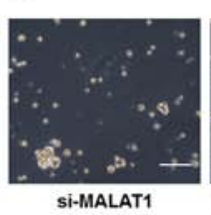
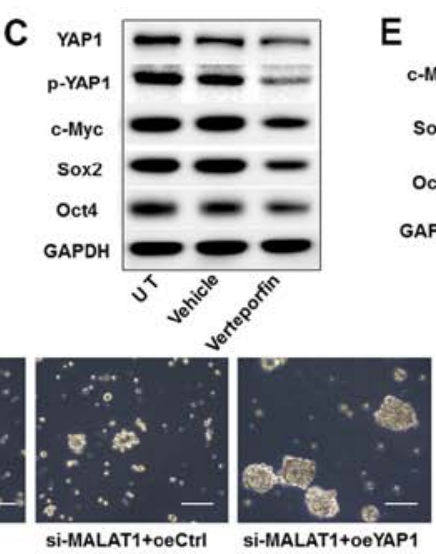

$\mathrm{E}$

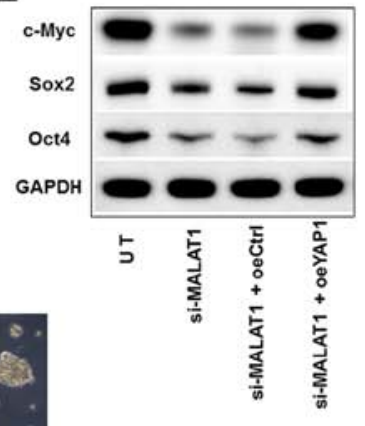

Figure 6. YAP1 mediates the regulation of MALAT1 on CSC features of liver cancer cells. (A) YAP1 overexpression in non-spherical Huh7 cells (box) or YAP1 suppression in spherical Huh7 cells (circle), followed by sphere formation assay (arrows). (B) At 2 weeks after sphere formation, UT, oeYAP1 and oeCtrl spheres of Huh7 cells were collected for Western blot analysis of expression of the stemness markers. (C) UT spheres, verteportin-treated spheres and vehicle-treated spheres from Huh7 cells were collected for Western blot analysis at 2 weeks after sphere formation. (D) Sphere formation assay and (E) Western blot analysis of expression of stemness factors on Huh7 cells transfected with si-MALAT1, or co-transfected with si-MALAT1 and oeYAP1 or oeCtrl. Scale bar, $100 \mu \mathrm{m}$. UT, untreated control cells. YAP1, Yes-associated protein 1; MALAT1, metastasis-associated lung adenocarcinoma transcript 1; CSC, cancer stem cell; oe, overexpression plasmid; Ctrl, control; si-, small interfering RNA; p-, phosphorylated.

CSC features of various tumors (21). Therefore, the present study further explored whether the modulatory effects of MALAT1 on CSC features were mediated by YAP1. As expected, YAP1 overexpression in non-sphere cells markedly promoted sphere formation (Fig. 6A), and increased the expression levels of c-Myc, Sox 2 and Oct4 (Fig. 6B). By contrast, verteporfin, a specific inhibitor for YAP1 expression and YAP1 phosphorylation inhibition (22), considerably impaired the sphere formation capacity of tumor cells (Fig. 6A), and reduced the expression levels of the corresponding stemness genes (Fig. 6C).

Subsequently, the role of YAP1 was investigated in MALAT1-modulated CSC properties. YAP1 overexpression rescued si-MALAT1-reduced sphere formation (Fig. 6D) and recovered the stemness factor expression levels in Huh7 cells (Fig. 6E). Therefore, these data indicated that MALAT1-mediated YAP1 signaling was required for the CSC features of liver cancer cells.

\section{Discussion}

Liver CSCs are one of the major factors contributing to the refractory nature of liver cancer to conventional treatment, and are closely associated with tumor growth, relapse, metastasis and chemoresistance $(4,23)$. Therefore, the successful development of potential therapeutic approaches that target liver CSCs is promising in improving the outcomes of patients with liver cancer. In the present study, it was revealed that the IncRNA MALAT1 was significantly increased in liver CSCs and that this lncRNA could maintain the stemness of liver CSCs by acting as a ceRNA for miR-375. This in turn regulated the expression of YAP1, which was functionally required for CSC attributes. The findings may contribute to liver cancer therapy by providing a new target for liver CSCs.

MALAT1 was one of the first lncRNAs shown to be involved in cancer progression, and a series of studies established the 
importance of MALAT1 as a cancer marker (24). MALAT1 upregulation has been observed in various cancer types, including lung, gastric, colorectal, prostate, melanoma, cervical, breast and liver cancer (25). In addition, MALAT1 upregulation may facilitate cell proliferation, migration, invasion and multidrug resistance of tumor cells, and predict the malignant progression and poor prognosis of liver cancer $(11,12,26)$. Recently, the role of MALAT1 in regulating the expression of stemness markers and CSC properties of cancer cells has attracted the attention of various researchers. In pancreatic cancer cells, MALAT1 knockdown reduced the formation of spheres and the expression of self-renewal related factors, such as Sox2, indicating that MALAT-1 may enhance stemness phenotypes of pancreatic CSCs by upregulating Sox 2 expression (13). The MALAT1-mediated induction of sphere formation and the upregulation of stemness biomarkers were also observed in osteosarcoma and glioma stem cells $(14,27)$. In the present study, MALAT1 was required for the CSC features of liver cancer cells, as determined by reduced sphere formation and sphere size, as well as decreased CSC-related factor expression, following siRNA-induced knockdown of MALAT1 expression levels.

lncRNAs act through various modes, including cotranscriptional regulation, gene expression modulation, nuclear or cytoplasmic complex scaffolding, and pairing with other RNAs (28). In addition, IncRNAs can exert oncogenic functions by mediating alternative splicing, and transcriptional and post-transcriptional regulation (29). One of the main mechanisms of MALAT1-mediated post-transcriptional regulation is its action as a ceRNA $(30,31)$. This mechanism suggests that lncRNAs act as molecular sponges for other RNA transcripts through the lncRNA-miRNA binding sites (also termed miRNA response elements) by competing for pool miRNAs $(30,31)$. MALAT1 has been shown to promote the growth, migration and invasion of liver cancer cells via the MALAT1/miR-195/epidermal growth factor receptor (11), MALAT1/miR-30a-5p/vimentin (32) and MALAT1/miR-143-3p/zinc finger E-box binding homeobox 1 axes (33), respectively. However, to the best of our knowledge, the exact role of MALAT1 in liver CSCs remains unknown. The present study further suggested the ceRNA role of MALAT1 in modulating the stemness of liver CSCs through the post-transcriptional regulation of YAP1 expression caused by sponging miR-375.

miR-375 was initially considered a pancreatic islet-specific miRNA that regulates insulin secretion, but was subsequently identified as a multifunctional miRNA with roles in pancreatic islet development, mucosal immunity, glucose homeostasis and even tumorigenesis (34). Previously, the significant reduction of miR-375 has been observed in multiple cancer types, including liver cancer $(35,36)$. Low miR-375 expression levels have been reported to be correlated with poor disease-free survival and may be a potential prognostic biomarker of disease progression in hepatocellular carcinoma (36). Further studies identified that miR-375 could suppress liver cancer cell proliferation and migration, and overcome chemoresistance by targeting several important oncogenes, such as JAK2, receptor tyrosine-protein kinase erbB-2, AEG-1 and autophagy-related 7 (37-40). Nanoparticle-mediated miR-375 delivery enhanced chemosensitivity and overcame multiple drug resistance in hepatocellular carcinoma, thus suggesting that miR-375 could be a potential target in liver cancer therapy (41). In the present study, a novel pathway was identified that explained the antitumor activity of miR-375 in liver cancer. MALAT1 knockdown may reverse sponging of miR-375, release miR-375 and boost its regulatory effects on oncogene expression at the post-transcriptional level.

In the current study, YAP1 was identified as a target of miR-375. YAP1 has an important role in the Hippo signaling pathway, which is an essential pathway in cell proliferation, apoptosis and organ growth regulation (42). YAP1 has also been reported to be involved in cancer development, since it can promote cell proliferation, suppress cell apoptosis, and induce the epithelial-mesenchymal transition and drug resistance of cancer cells (43). An increasing number of studies have determined the role of YAP1 in modulating cellular stemness. YAP1 overexpression may induce CSC properties, such as self-renewal, sphere-formation, invasiveness and drug resistance $(21,44)$. Further studies showed that these YAP1-related CSC biological features were mediated by regulating embryonic stem cell factors, such as Oct4, Sox 2 and Nanog $(45,46)$. The results of the present study were consistent with the observations that YAP1 overexpression promoted sphere formation, and upregulated Sox 2 and Oct 4 expression levels. MALAT1 knockdown-induced suppression of sphere formation and the corresponding reduction in expression of stemness markers could also be rescued by YAP1 overexpression, suggesting that MALAT1 contributed to CSC properties via YAP1. These observations provide information regarding the contribution of MALAT1 to CSC-associated biological features via the competitive regulation of miR-375-mediated YAP1 expression.

In summary, the present study demonstrated that MALAT1 was highly expressed in liver CSCs and that it promoted the CSC properties in cell culture by upregulating YAP1 expression via sponging miR-375. These findings elucidated the potential role of the MALAT1/miR-375/YAP1 axis in modulating the stemness of liver cancer cells and improved our understanding of the molecular characteristics of liver CSCs. The current study lays the foundation for the development of novel therapeutic strategies to eradicate CSCs that are potentially harbored in residual cancerous liver lesions.

\section{Acknowledgements}

Not applicable.

\section{Funding}

This work was supported by the general research project of education of Zhejiang province (grant no. Y201738069), the National Natural Science Fund of China (grant no. 81870428), and the Key R\&D Projects of Zhejiang Province (grant no. 2018C03019).

\section{Availability of data and materials}

The datasets used and/or analyzed during the current study are available from the corresponding author on reasonable request. 


\section{Authors' contributions}

LZ and GL performed the research and drafted the manuscript. AL participated in in vitro studies and performed the statistical analysis. YL conceived the study, and participated in its design and coordination. All authors read and approved the final manuscript.

\section{Ethics approval and consent to participate}

The present study was approved by The Second Affiliated Hospital, Zhejiang University Hospital Ethics Committee (ethics no. 2019-099), and all patients signed informed consent for their participation in the study.

\section{Patient consent for publication}

Not applicable.

\section{Competing interests}

The authors declare that they have no competing interests.

\section{References}

1. Ferlay J, Soerjomataram I, Dikshit R, Eser S, Mathers C Rebelo M, Parkin DM, Forman D and Bray F: Cancer incidence and mortality worldwide: Sources, methods and major patterns in GLOBOCAN 2012. Int J Cancer 136: E359-E386, 2015.

2. Bruix J, Gores GJ and Mazzaferro V: Hepatocellular carcinoma: Clinical frontiers and perspectives. Gut 63: 844-855, 2014.

3. Prager BC, Xie Q, Bao S and Rich JN: Cancer stem cells: The architects of the tumor ecosystem. Cell Stem Cell 24: 41-53, 2019

4. Najafi M, Farhood B and Mortezaee K: Cancer stem cells (CSCs) in cancer progression and therapy. J Cell Physiol 234: 8381-8395, 2019.

5. Xiang Y, Yang T, Pang BY, Zhu Y and Liu YN: The progress and prospects of putative biomarkers for liver cancer stem cells in hepatocellular carcinoma. Stem Cells Int 2016: 7614971, 2016.

6. Wang N, Wang S, Li MY, Hu BG, Liu LP, Yang SL, Yang S, Gong Z, Lai PBS and Chen GG: Cancer stem cells in hepatocellular carcinoma: An overview and promising therapeutic strategies. Ther Adv Med Oncol 10: 1758835918816287, 2018.

7. Yamashita T, Honda M, Nakamoto $\mathrm{Y}$, Baba M, Nio K, Hara Y, Zeng SS, Hayashi T, Kondo M, Takatori H, et al: Discrete nature of $\mathrm{EpCAM}^{+}$and $\mathrm{CD}^{+}$cancer stem cells in human hepatocellular carcinoma. Hepatology 57: 1484-1497, 2013.

8. Batista PJ and Chang HY: Long noncoding RNAs: Cellular address codes in development and disease. Cell 152: 1298-1307, 2013.

9. Sanchez Calle A, Kawamura Y, Yamamoto Y, Takeshita F and Ochiya T: Emerging roles of long non-coding RNA in cancer. Cancer Sci 109: 2093-2100, 2018.

10. Zhang X, Hamblin $\mathrm{MH}$ and Yin KJ: The long noncoding RNA Malat1: Its physiological and pathophysiological functions. RNA Biol 14: 1705-1714, 2017.

11. Liu D, Zhu Y, Pang J, Weng X, Feng X and Guo Y: Knockdown of long non-coding RNA MALAT1 inhibits growth and motility of human hepatoma cells via modulation of miR-195. J Cell Biochem 119: 1368-1380, 2018.

12. Yuan P, Cao W, Zang Q, Li G, Guo X and Fan J: The HIF-2 $\alpha$-MALAT1-miR-216b ax is regulates multi-drug resistance of hepatocellular carcinoma cells via modulating autophagy. Biochem Biophys Res Commun 478: 1067-1073, 2016.

13. Jiao F, Hu H, Han T, Yuan C, Wang L, Jin Z, Guo Z and Wang L: Long noncoding RNA MALAT-1 enhances stem cell-like phenotypes in pancreatic cancer cells. Int J Mol Sci 16: 6677-6693, 2015.

14. Han Y, Zhou L, Wu T, Huang Y, Cheng Z, Li X, Sun T, Zhou Y and DuZ: Downregulation of IncRNA-MALAT1 affects proliferation and the expression of stemness markers in glioma stem cell line SHG139S. Cell Mol Neurobiol 36: 1097-1107, 2016.
15. Livak KJ and Schmittgen TD: Analysis of relative gene expression data using real-time quantitative PCR and the 2(-Delta Delta $\mathrm{C}(\mathrm{T})$ ) method. Methods 25: 402-408, 2001.

16. Akita H, Marquardt JU, Durkin ME, Kitade M, Seo D, Conner EA, Andersen JB, Factor VM and Thorgeirsson SS: MYC activates stem-like cell potential in hepatocarcinoma by a p53-dependent mechanism. Cancer Res 74: 5903-5913, 2014.

17. Leis O, Eguiara A, Lopez-Arribillaga E, Alberdi MJ, HernandezGarcia S, Elorriaga K, Pandiella A, Rezola R and Martin AG: Sox 2 expression in breast tumours and activation in breast cancer stem cells. Oncogene 31: 1354-1365, 2012.

18. Kuo KK, Lee KT, Chen KK, Yang YH, Lin YC, Tsai MH, Wuputra K, Lee YL, Ku CC, Miyoshi H, et al: Positive feedback loop of OCT4 and c-JUN expedites cancer stemness in liver cancer. Stem Cells 34: 2613-2624, 2016.

19. Fu H, Fu L, Xie C, Zuo WS, Liu YS, Zheng MZ and Yu JM: miR-375 inhibits cancer stem cell phenotype and tamoxifen resistance by degrading HOXB3 in human ER-positive breast cancer. Oncol Rep 37: 1093-1099, 2017.

20. Zhu P, Wang Y, Wu J, Huang G, Liu B, Ye B, Du Y, Gao G, Tian Y, He L, et al: LncBRM initiates YAP1 signalling activation to drive self-renewal of liver cancer stem cells. Nat Commun 7: 13608, 2016.

21. Zanconato F, Cordenonsi M and Piccolo S: YAP/TAZ at the Roots of Cancer. Cancer Cell 29: 783-803, 2016.

22. Kandasamy S, Adhikary G, Rorke EA, Friedberg JS, Mickle MB, Alexander HR and Eckert RL: The YAP1 signaling inhibitors, verteporfin and CA3, suppress the mesothelioma cancer stem cell phenotype. Mol Cancer Res 18: 343-351, 2020.

23. Nio K, Yamashita T and Kaneko S: The evolving concept of liver cancer stem cells. Mol Cancer 16: 4, 2017.

24. Li ZX, Zhu QN, Zhang HB, Hu Y, Wang G and Zhu YS: MALAT1: A potential biomarker in cancer. Cancer Manag Res 10: 6757-6768, 2018.

25. Yoshimoto R, Mayeda A, Yoshida M and Nakagawa S: MALAT1 long non-coding RNA in cancer. Biochim Biophys Acta 1859: 192-199, 2016.

26. Konishi H, Ichikawa D, Yamamoto Y, Arita T, Shoda K, Hiramoto H, Hamada J, Itoh H, Fujita Y, Komatsu S, et al: Plasma level of metastasis-associated lung adenocarcinoma transcript 1 is associated with liver damage and predicts development of hepatocellular carcinoma. Cancer Sci 107: 149-154, 2016.

27. Chen Y, Huang W, Sun W, Zheng B, Wang C, Luo Z, Wang J and Yan W: LncRNA MALAT1 promotes cancer metastasis in osteosarcoma via activation of the PI3K-Akt signaling pathway. Cell Physiol Biochem 51: 1313-1326, 2018.

28. Ulitsky I and Bartel DP: lincRNAs: Genomics, evolution, and mechanisms. Cell 154: 26-46, 2013.

29. Kondo Y, Shinjo K and Katsushima K: Long non-coding RNAs as an epigenetic regulator in human cancers. Cancer Sci 108: 1927-1933, 2017.

30. Tay Y, Rinn J and Pandolfi PP: The multilayered complexity of ceRNA crosstalk and competition. Nature 505: 344-352, 2014.

31. Wang WT, Ye H, Wei PP, Han BW, He B, Chen ZH and Chen YQ: LncRNAs H19 and HULC, activated by oxidative stress, promote cell migration and invasion in cholangiocarcinoma through a ceRNA manner. J Hematol Oncol 9: 117, 2016.

32. Pan Y, Tong S, Cui R, Fan J, Liu C, Lin Y, Tang J, Xie H, Lin P, Zheng T, et al: Long non-coding MALAT1 functions as a competing endogenous RNA to regulate vimentin expression by sponging miR-30a-5p in hepatocellular carcinoma. Cell Physiol Biochem 50: 108-120, 2018.

33. Chen L, Yao H, Wang $\mathrm{K}$ and Liu X: Long non-coding RNA MALAT1 regulates ZEB1 expression by sponging miR-143-3p and promotes hepatocellular carcinoma progression. J Cell Biochem 118: 4836-4843, 2017.

34. Li X: MiR-375, a microRNA related to diabetes. Gene 533: 1-4, 2014.

35. Yan JW, Lin JS and He XX: The emerging role of miR-375 in cancer. Int J Cancer 135: 1011-1018, 2014.

36. Zhou N, Wu J, Wang X, Sun Z, Han Q and Zhao L: Low-level expression of microRNA-375 predicts poor prognosis in hepatocellular carcinoma. Tumour Biol 37: 2145-2152, 2016.

37. Cao S, Wang G, Wang J, Li C and Zhang L: Hsa circ 101280 promotes hepatocellular carcinoma by regulating miR-375/ JAK2. Immunol Cell Biol 97: 218-228, 2019.

38. Li L, Jia L and Ding Y: Upregulation of miR-375 inhibits human liver cancer cell growth by modulating cell proliferation and apoptosis via targeting ErbB2. Oncol Lett 16: 3319-3326, 2018. 
39. He XX, Chang Y, Meng FY, Wang MY, Xie QH, Tang F, Li PY, Song YH and Lin JS: MicroRNA-375 targets AEG-1 in hepatocellular carcinoma and suppresses liver cancer cell growth in vitro and in vivo. Oncogene 31: 3357-3369, 2012.

40. Chang Y, Yan W, He X, Zhang L, Li C, Huang H, Nace G, Geller DA, Lin J and Tsung A: miR-375 inhibits autophagy and reduces viability of hepatocellular carcinoma cells under hypoxic conditions. Gastroenterology 143: 177-87.e8, 2012.

41. Zhao P, Wu S, Cheng Y, You J, Chen Y, Li M, He C, Zhang X, Yang T, Lu Y, et al: MiR-375 delivered by lipid-coated doxorubicin-calcium carbonate nanoparticles overcomes chemoresistance in hepatocellular carcinoma. Nanomedicine (Lond) 13: 2507-2516, 2017.

42. Hansen CG, Moroishi T and Guan KL: YAP and TAZ: A nexus for Hippo signaling and beyond. Trends Cell Biol 25: 499-513, 2015.

43. Shibata M, Ham K and Hoque MO: A time for YAP1: Tumorigenesis, immunosuppression and targeted therapy. Int J Cancer 143: 2133-2144, 2018.
44. Lu T, Li Z, Yang Y, Ji W, Yu Y, Niu X, Zeng Q, Xia W and Lu S: The Hippo/YAP1 pathway interacts with FGFR1 signaling to maintain stemness in lung cancer. Cancer Lett 423: 36-46, 2018.

45. Bora-Singhal N, Nguyen J, Schaal C, Perumal D, Singh S, Coppola D and Chellappan S: YAP1 regulates OCT4 activity and SOX2 expression to facilitate self-renewal and vascular mimicry of stem-like cells. Stem Cells 33: 1705-1718, 2015.

46. Strnadel J, Choi S, Fujimura K, Wang H, Zhang W, Wyse M, Wright T, Gross E, Peinado C, Park HW, et al: eIF5A-PEAK1 signaling regulates YAP1/TAZ protein expression and pancreatic cancer cell growth. Cancer Res 77: 1997-2007, 2017.

This work is licensed under a Creative Commons

Attribution-NonCommercial-NoDerivatives 4.0 International (CC BY-NC-ND 4.0) License. 